\title{
NUMERICAL EVALUATION OF THE GAUSS HYPERGEOMETRIC FUNCTION BY POWER SUMMATIONS
}

\author{
JURGEN A. DOORNIK
}

Abstract. Numerical evaluation of the Gauss hypergeometric function ${ }_{2} F_{1}(a, b ; c ; z)$, with complex parameters $a, b, c$ and complex argument $z$ is notoriously difficult. Carrying out the summation that defines the function may fail, even for moderate values of $z$. Formulae are available to transform the effective argument in the series, potentially leading to a numerically successful summation. Unfortunately, these transformations have a singularity when $b-a$ or $c-a-b$ is an integer, and suffer numerical instability near that. This singularity has to be removed analytically after collecting powers in $z$.

The contributions in this paper are fourfold. First, analytical expressions are provided that remove the singularity from Bühring's $1 /\left(z-z_{0}\right)$ transformation. This is more difficult, because the singularity occurs twice, and it is necessary to collect powers of $z_{0}$, as well as $z$. The resulting expression has a three-term recursion, like the original. Next, improved expressions are derived for the cases that have been addressed before. We study a transformation that converges outside $|z-0.32|>0.32$ for $\mathcal{R} z>0$, which is tighter than the $|z-0.5|>0.5$ which is normally considered. Finally, we derive an improved algorithm for the numerical evaluation of ${ }_{2} F_{1}$.

\section{INTRODUCTION}

The Gauss hypergeometric function is defined as

$$
{ }_{2} F_{1}(a, b ; c ; z)=\sum_{i=0}^{\infty} \frac{(a)_{i}(b)_{i}}{(c)_{i} i !} z^{i} \equiv F(z)
$$

for the argument $|z|<1$ and parameters $a, b \in \mathbb{C}, c \in \mathbb{C} \backslash\{0,-1,-2, \ldots\}$. The hypergeometric function is extended to $z \in \mathbb{C}$ by analytic continuation. The function does not exist when $c=0$ or for $c$ a negative integer (unless $a$ or $b$ is a smaller negative integer or zero). The principal branch corresponds to $|\operatorname{ph}(1-z)| \leq \pi$. The notation in (11) uses the Pochhammer symbol:

$$
(a)_{i}=a(a+1)(a+2) \ldots(a+i-1), \quad(a)_{0}=1 .
$$

The Pochhammer symbol can be expressed as a ratio of gamma functions: $(a)_{i}=$ $\Gamma(a+i) / \Gamma(a)$, provided $a$ is not zero or a negative integer. The following notation is used throughout:

$$
\frac{\Gamma(a) \Gamma(b)}{\Gamma(c) \Gamma(d)}=\Gamma\left(\begin{array}{l}
a, b \\
c, d
\end{array}\right)
$$

Received by the editor May 1, 2013 and, in revised form, September 25, 2013 and October 25, 2013.

2010 Mathematics Subject Classification. Primary 33C05, 65D20.

Key words and phrases. Gauss hypergeometric function.

This research was supported in part by grants from the Open Society Foundations and the Oxford Martin School. 
The complex $z$ is also expressed as $z=z_{r}+\mathbf{i} z_{i}=\mathcal{R} z+\mathbf{i} \mathcal{I} z$; while $i$ is used as a summation index. We shall write $|\mathcal{R}(a, b, c)| \leq \delta$ as shorthand for $|\mathcal{R} a| \leq \delta$, $|\mathcal{R} b| \leq \delta$, and $|\mathcal{R} c| \leq \delta$. Similar notation is used for $|\mathcal{I}(a, b, c)|$.

On the unit circle $|z|=1$, the Gauss hypergeometric function converges absolutely when $\mathcal{R}(c-a-b)>0$, converges conditionally when $-1<\mathcal{R}(c-a-b) \leq 0$ (except for $z=1$ ), and diverges otherwise; 1, Ch. 15] and [10] provide more information.

When $z$ is small, the hypergeometric function can be directly evaluated using (11) until the successive terms get sufficiently small. For larger $z$, the summation may converge very slowly or not at all. Negative values for $a, b$ or $c$ can create additional problems.

Forrey [5] adopts transformation formulae to extend the applicability of the summation: for real parameters and real argument only, the space of $z$ is partioned in six regions: $(-\infty,-1),[-1,0),[0,0.5],(0.5,1],(1,2],(2, \infty)$. The transformations used are, respectively, $1 /(1-z), z /(z-1), z, 1-z, z /(z-1), 1 / z$. A major complication is that, except for $z /(z-1)$, the transformation formulae consist of two diverging terms for certain values of the parameters $a, b, c$, even though the overall result is well defined. In practice this causes severe numerical instability close to those values. Forrey in [5] uses finite differences to resolve these issues, and evaluates the proposed algorithm against some special cases of ${ }_{2} F_{1}$ for which analytical solutions are known.

Skorokhodov 11] gives a detailed analysis of these difficult cases for complex parameters and arguments. Several algorithms are given for the function

$$
\Gamma_{\epsilon}(z)=\frac{1}{\epsilon}\left[\Gamma\left(\begin{array}{c}
z+\epsilon \\
z
\end{array}\right)-1\right],
$$

which must be computed accurately for small $\epsilon$ as part of avoiding the numerical problems.

Michel and Stoitsov 9 also extend the computation of the hypergeometric function to complex arguments, independently of [11. They study the performance of their algorithm for a wide range of argument values when the parameters are small, and provide computer code. As [6] and [12, §2.2] point out, selecting

$$
|z| \leq \rho,|1-z| \leq \rho,\left|\frac{1}{z}\right| \leq \rho,\left|\frac{z-1}{z}\right| \leq \rho,\left|\frac{1}{1-z}\right| \leq \rho,\left|\frac{z}{z-1}\right| \leq \rho,
$$

with $0<\rho<1$, leaves two gaps around $\exp ( \pm \pi \mathbf{i} / 3)$. Michel and Stoitsov [9] fill this with a Taylor series expansion.

Bühring 2] obtained a three-term recurrence that implements the $1 /\left(z-z_{0}\right)$ transformation. This is found to be important below, because, with a judicious choice of $z_{0}$, it can succeed in areas where other summations perform poorly, or fail. Removing the singularity is harder, because it appears twice in a double summation, requiring first to collect powers in $z$, then in $z_{0}$. The novel expression is again a three-term recurrence.

The remainder of this paper is organized as follows. Section 2 lists some of the basic results that are used, and briefly introduces the algorithms. This is followed by implementation and evaluation of the complex case in $₫ 3$.

All analytical derivations are contained in the Appendix. The focus is on removing the singularities that arise in the linear transformations, and defining some

\footnotetext{
${ }^{1} \mathrm{I}$ am grateful to an anonymous referee for providing this reference.
} 
auxiliary functions that are used. Good implementations of the $\Gamma, \log \Gamma$, digamma $\psi$, and polygamma functions $\left(\psi^{\prime}, \psi^{\prime \prime}\right)$ are required. A.1 removes the singularity from the $1-z$ transformation that occurs when $c-a-b$ is an integer. The real version is also considered. The $1 / z$ transformation, $\mathrm{A} .2$, has a singularity when $b-a$ is an integer. The resulting expression resembles the $1-z$ expressions. A.3 handles the $1 /\left(z-z_{0}\right)$ transformation.

It is useful to treat the ratio of gamma functions (2) as a separate function to handle cancellations. Appendix B defines how this function is evaluated, as well as $\Gamma_{\epsilon}(z), \operatorname{sinc}(z)=\sin (\pi z) / \pi z$ and $E_{\epsilon}(z)=[\exp (\epsilon z)-1] / \epsilon$.

\section{Algorithmic FrameWork}

2.1. Special values. The following special values are handled directly:

\begin{tabular}{ccc}
\hline special value & ${ }_{2} F_{1}(a, b ; c ; z)$ & condition \\
\hline$z=0$ & 1 & \\
$z=1$ & $\Gamma\left(\begin{array}{c}c, c-a-b \\
c-a, c-b\end{array}\right)$ & $\mathcal{R}(c-a-b)>0, c \neq-m$ \\
$c=b$ & $(1-z)^{-a}$ & \\
$c=a$ & $(1-z)^{-b}$ & \\
$c=a-1$ & $(1-z+b z / c)(1-z)^{-1-b}$ & \\
$c=b-1$ & $(1-z+a z / c)(1-z)^{-1-a}$ & \\
\hline
\end{tabular}

2.2. Linear transformations. Several linear transformations are considered. The first two only affect the parameters in the numerator:

$$
\begin{aligned}
{ }_{2} F_{1}(a, b ; c ; z) & ={ }_{2} F_{1}(b, a ; c ; z) \\
& =(1-z)^{c-a-b}{ }_{2} F_{1}(c-a, c-b ; c ; z) \\
& =(1-z)^{-a}{ }_{2} F_{1}\left(a, c-b ; c ; \frac{z}{z-1}\right) \\
& =(1-z)^{-b}{ }_{2} F_{1}\left(c-a, b ; c ; \frac{z}{z-1}\right) .
\end{aligned}
$$

Further expressions are available to transform $z$ :

$$
\begin{gathered}
{ }_{2} F_{1}(a, b ; c ; z)=\Gamma\left(\begin{array}{c}
c,-d \\
a, b
\end{array}\right)(1-z)_{2}^{d} F_{1}(c-a, c-b ; 1+d ; 1-z) \\
+\Gamma\left(\begin{array}{c}
c, d \\
c-a, c-b
\end{array}\right){ }_{2} F_{1}(a, b ; 1-d ; 1-z), \quad d=c-a-b \\
=\Gamma\left(\begin{array}{c}
c,-d \\
a, c-b
\end{array}\right)(-z)^{-b}{ }_{2} F_{1}\left(b, 1-c+b ; 1+d ; \frac{1}{z}\right) \\
+\Gamma\left(\begin{array}{c}
c, d \\
b, c-a
\end{array}\right)(-z)^{-a}{ }_{2} F_{1}\left(a, 1-c+a ; 1-d ; \frac{1}{z}\right), \quad d=b-a \\
=\Gamma\left(\begin{array}{c}
c,-d \\
a, c-b
\end{array}\right)\left(-z+z_{0}\right)^{-b} \sum_{i=0}^{\infty} f_{i}\left(z_{0}\right)\left(z-z_{0}\right)^{-i} \\
+\Gamma\left(\begin{array}{c}
c, d \\
b, c-a
\end{array}\right)\left(-z+z_{0}\right)^{-a} \sum_{i=0}^{\infty} g_{i}\left(z_{0}\right)\left(z-z_{0}\right)^{-i}, \quad d=b-a
\end{gathered}
$$

The $1-z$ transformation (8) has a pole when $c-a-b= \pm m$, and may break down when close to that, see $\$$ A.1. The $1 / z$ transformation (9) and $1 /\left(z-z_{0}\right)$ 
transformation (10) have a pole when $b-a= \pm m$, and may break down when close to that; see $\$$ A.2 and $\$$ A.3 respectively. The transformation (10) was obtained in [2]; $f_{i}\left(z_{0}\right)$ and $g_{i}\left(z_{0}\right)$ are three-term recurrences defined in $\$$ A.3.

2.3. Assessing numerical accuracy. Numerical accuracy is evaluated along the lines proposed in $9, \S 7.1]$, measuring how closely the solution satisfies the hypergeometric differential equation

$$
\begin{aligned}
& T=\frac{\left|z(1-z) f_{2}+[c-(a+b+1) z] f_{1}-F(z)\right|}{|F(z)|+\left|f_{1}\right|+\left|f_{2}\right|}, \\
& f_{1}=\frac{1}{c} F(+1 ; z), f_{2}=\frac{(a+1)(b+1)}{c(c+1)} F(+2 ; z),
\end{aligned}
$$

where $F(+n ; z)$ is shorthand for ${ }_{2} F_{1}(a+n, b+n ; c+n ; z)$. Parameter values $a, b, c$ and argument $z$ are sampled randomly from a specified interval, each time evaluating (11). From this we compute the average accuracy $T_{\text {avg }}$ and the worst case accuracy $T_{\max }$. The reported numbers are $t_{\mathrm{avg}}=-\log _{10} T_{\mathrm{avg}}$, which roughly correspond to the number of correctly computed significant digits (this accuracy is relative, not absolute). When there are no correct digits the accuracy is reported as zero (in that case the average accuracy is meaningless). An accuracy of 15 corresponds roughly to 15 significant digits, close to the double-precision machine precision of around $10^{-16}$. Double precision is used throughout.

2.4. Basic algorithm. The basic algorithm is the direct summation (11) without applying any transformation. The summation terminates when 2

(1) the maximum number of iterations is reached, or

(2) when the following conditions hold jointly:

(a) the minimum number of iterations is reached; and

(b) two successive iterations have $|t|_{\infty} \leq 10^{-15}|F(z)|_{\infty}$, where $t$ is the increment.

(3) The summation fails when the maximum of 10000 iterations is reached.

The minimum number of iterations is only set when any of $\mathcal{R}(a, b, c)$ is negative or zero. In that case it is set to three minus the integer part of the smallest value. This ensures that termination cannot happen until the real parts of $a+i, b+i, c+i$ are all positive (with $i$ the loop counter), and is a convenient alternative to estimating the required number of iterations. When $a$ and/or $b$ are exactly equal to zero or a negative integer, this determines the number of iterations.

A pre-processing stage detects whether any of $a, b, c-a, c-b$ are an integer, in which case the basic algorithm is always used.

2.5. Choosing the sign of $\mathcal{R}(c-a-b)$ in the basic algorithm. Michel and Stoitsov [9, §7.1] argue that transformation (5) should be applied whenever $\mathcal{R} d=$ $\mathcal{R}(c-a-b)<0$ because the transformation is numerically stable and (1) behaves as $z^{i} / i^{c-a-b}$ for $i \rightarrow \infty$. We follow this advice, but note that there are situations where slow convergence is numerically beneficial: Table 1 gives two examples with negative $c$.

\footnotetext{
${ }^{2}$ We do not pre-estimate the number of iterations, as this is fairly costly, and unnecessary.
} 
TABLE 1. Accuracy of some hypergeometric functions using summation (11), comparing transformation to $d=c-a-b<0$ with transformation to $d^{*}=c-a^{*}-b^{*}>0$, where $a^{*}=c-b, b^{*}=c-a$.

\begin{tabular}{cccc|cc|cc}
\hline & & & & \multicolumn{2}{|c|}{$(\mathbf{1 1}): \mathcal{R} d<0$} & \multicolumn{2}{|c}{ (5) and (1) $: \mathcal{R} d^{*}>0$} \\
& & & & ${ }_{2} F_{1}(a, b ; c ; z)$ & \multicolumn{2}{c}{$(1-z)_{2}{ }_{2} F_{1}\left(a^{*}, b^{*} ; c ; z\right)$} \\
$a$ & $b$ & $c$ & $z$ & $-\log _{10} T$ & iterations & $-\log _{10} T$ & iterations \\
\hline-12.9 & 16.2 & -10.4 & 0.9 & 15.8 & 587 & 2.7 & 129 \\
-7.9 & 11.2 & -5.4 & 0.9 & 16.3 & 492 & 7.0 & 144 \\
\hline
\end{tabular}

2.6. Algorithms for evaluating ${ }_{2} F_{1}(a, b ; c ; z)$. If necessary, $a$ and $b$ are swapped to achieve $\mathcal{R} a \leq \mathcal{R} b$. The following algorithms are evaluated:

$A[z]$ : Uses the direct summation $\$ 2.4$ after imposing $\mathcal{R}(c-a-b)>0$ using (5).

$A[z /(z-1)]$ : The $z /(z-1)$ transformation (6) is used. If we denote the parameters and argument in the transformed function by an asterisk, we now need to compute ${ }_{2} F_{1}\left(a^{*}, b^{*} ; c^{*} ; z^{*}\right)$, where $a^{*}=a, b^{*}=c-b, c^{*}=$ $c, z^{*}=z /(z-1)$. So we automatically have $\mathcal{R} d^{*} \geq 0$ because $d^{*}=b-a$, and we already have $\mathcal{R} a \leq \mathcal{R} b$ from (4). This is followed by the direct summation $\$ 2.4$

$A[1-z]$ : Implementation of the $1-z$ expression (8) requires that (near-) singularity when $c-a-b$ is (close to) integer is analytically removed. This is done for $\mathcal{R} d>0$ in A.1. where $d=c-a-b$. A related approach can be used for $\mathcal{R} d<0$, but avoided here through (5).

$A[1 / z]$ : Expression (5) is applied to get the resulting $\mathcal{R}(c-a-b)>0$, if needed. A.2 documents how $b-a$ near integer is handled.

The approach to removing the poles in $A[1-z]$ and $A[1 / z]$ is similar to [1] and [9, but providing more attractive expressions, in particular for the latter case.

Unlike [5], we take finite difference approximations of $\Gamma(z+\epsilon) / \Gamma(z)$ rather than $\Gamma(z+\epsilon)-\Gamma(z)$. Assuming that the polygamma function is available, the resulting expressions are quite simple. Central differences are used to achieve higher accuracy for the same computational effort; see $₫ \mathrm{~B} .2$.

$A[(z-1) / z]$ : The $z /(z-1)$ transformation (6) is used with $A[1 / z]$.

$A[1 /(1-z)]$ : Combines (6) and $A[1-z]$ to implement the $1 /(1-z)$ transformation.

$A[1 /(2 z-1)]$ : This is based on the implementation of the $1 /\left(z-z_{0}\right)$ expression (10), which converges outside the circle $\left|z-z_{0}\right|=\max \left(\left|z_{0}\right|,\left|1-z_{0}\right|\right)$; see 2. The approach to removing the singularity is given in $\$$ A.3. One of the summations is a three-term recurrence, and, as a result, the algorithm is about half as slow as $A[1 / z]$. On the other hand, the presence of $z_{0}$ allows us to evaluate the hypergeometric function where other algorithms fail. Here we use $z_{0}=1 / 2$, to give convergence outside the circle $|z-1 / 2|=1 / 2$. So we can treat it as the additional transformation:

$$
|1 /(2 z-1)| \leq \rho<1
$$


TABLE 2. Values of $\rho$ for similar accuracy of standard algorithms.

\begin{tabular}{lccc}
\hline Algorithm & transformation & $\begin{array}{c}t_{\text {avg }} \approx 12 \\
\rho=\rho_{1}\end{array}$ & $\begin{array}{c}t_{\text {avg }} \approx 10 \\
\rho=\rho_{2}\end{array}$ \\
\hline$A[z]$ & $|z| \leq \rho$ & 0.9 & 0.95 \\
$A[z /(z-1)]$ & $|z /(z-1)| \leq \rho$ & 0.9 & 0.95 \\
$A[1-z]$ & $|1-z| \leq \rho$ & 0.6 & 0.9 \\
$A[1 /(1-z)]$ & $|1 /(1-z)| \leq \rho$ & $1 / 3$ & 0.5 \\
$A[1 / z]$ & $|1 / z| \leq \rho$ & $1 / 3$ & 0.5 \\
$A[(z-1) / z]$ & $|(z-1) / z| \leq \rho$ & 0.5 & 0.7 \\
\hline
\end{tabular}

$A[(z-1) /(z+1)]$ : The $z /(z-1)$ transformation (6) is used with $A[1 /(2 z-1)]$. The additional transformation is:

$$
|(z-1) /(z+1)| \leq \rho<1 .
$$

$A[z /(z-1)-1 /(2 z)]$ : The previous two algorithms used the $1 /\left(z-z_{0}\right)$ expression (10) with $z_{0}=0.5$, as is customary in the literature.

Another interesting case is $z_{0}=0.5(1-1 / z)$ together with $z /(z-1)$ to give the transformation:

$$
\left(\frac{z}{z-1}-\frac{1}{2 z}\right)^{-1}
$$

For $\mathcal{R} z>0$ this converges for

$$
|z-\alpha|>\alpha, \text { where } \alpha=\frac{1+\sqrt{17}}{16} \approx 0.32,
$$

which is considerably better than $|z-0.5|>0.5$. Some numerical comparisons with $A[1 /(2 z-1)]$ are provided below.

\section{Numerical EVAluation}

We consider moderate parameter values, which are taken as both $|\mathcal{R}(a, b, c)| \leq 4$ and $|\mathcal{I}(a, b, c)| \leq 4$.

Numerical experiments were performed for the standard transformations as embodied by the circles (3), for expanding values of $\rho$. We use the algorithms listed in 2.6 , initially excluding those based on $1 /\left(z-z_{0}\right)$, and bounding the randomly selected parameter values away from integer values. The first observation is that different algorithms achieve similar accuracy for very different values of $\rho$.

If we require about 12 accurate digits on average, and no worse than about 8 , we find approximate cut-off values as reported in the $\rho_{1}$ column of Table2. The circles are extended to $\rho_{2}$ if we accept about 10 accurate digits and no worse than 6 . The circles for $\rho_{1}$ are displayed in the left panel of Figure 1] showing a substantial area of the $z$ space not yet covered. The right panel of Figure 1 shows a smaller, but still substantial, uncovered area when adopting the $\rho_{2}$ values.

The left panel of Figure 1 has $\exp ( \pm \pi \mathbf{i} / 3)$ marked by black dots. This is only just outside the accurate region, and could be handled by a Taylor series expansion, as in 9 .

The $1 /\left(z-z_{0}\right)$ transformation provides a tighter region of convergence, which could fill the gaps around $\exp ( \pm \pi \mathbf{i} / 3)$. Unfortunately, it appears that most of this 

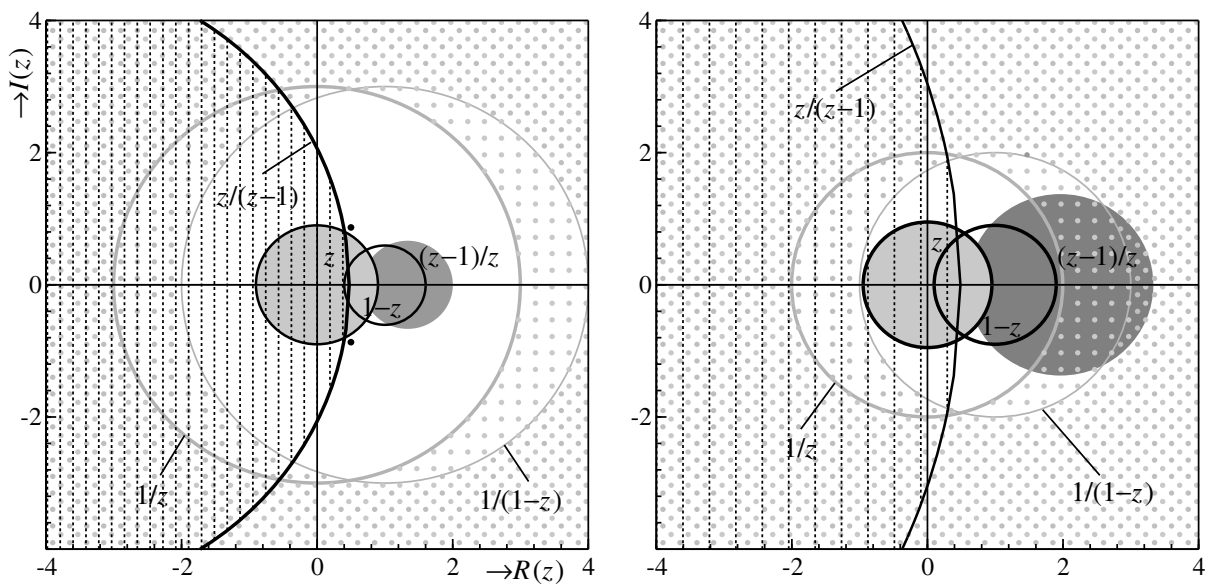

FiguRE 1. Coverage obtained by standard algorithms for similar accuracy. Left panel: circular regions of high accuracy (about 12 digits). Right panel: regions with reduced accuracy (about 10 digits).
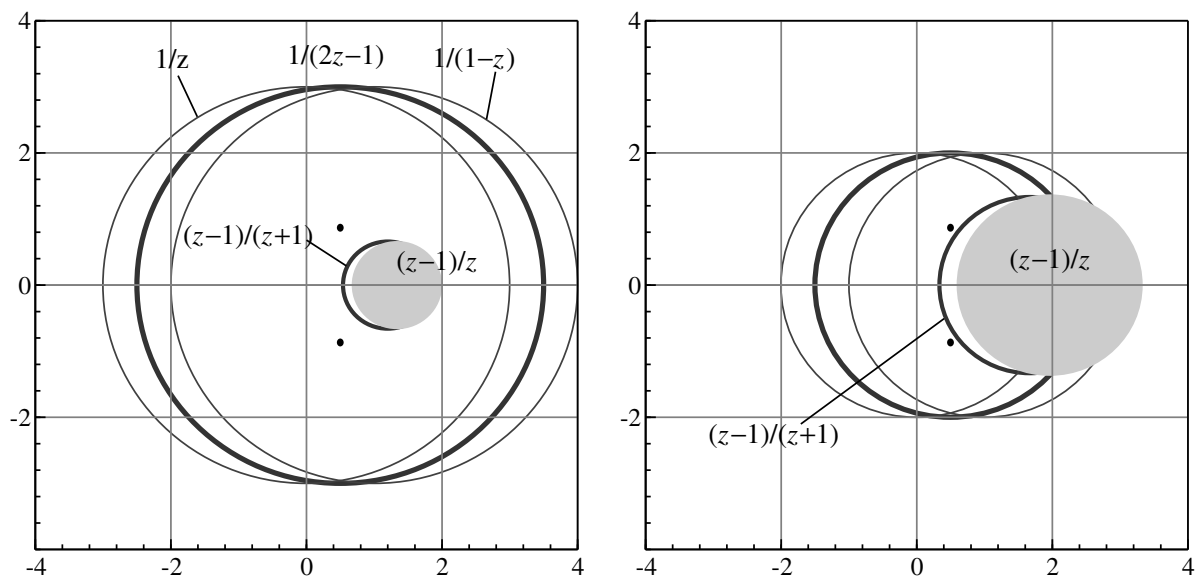

Figure 2. Accuracy with small parameters for $1 /\left(z-z_{0}\right)$ transformations for $z_{0}=0.5$. Left panel: circular regions of high accuracy (about 12 digits). Right panel: regions with reduced accuracy (about 10 digits).

advantage is lost again when taking the accuracy of $A[1 /(2 z-1)]$ and $A[(z-1) /(z+$ $1)]$ into account. Both panels of Figure 2 show that, for the same accuracy, the area outside $1 /(2 z-1)$ is encompassed by the area outside either $1 /(1-z)$ or $1 / z$. Of course, the $1 /(2 z-1)$ transformation can still reach where the other two cannot, but at a low average of accuracy. The $(z-1) /(z+1)$ transformation has the same radius as $(z-1) / z$ for similar accuracy. There is a small gain to the left of the solid gray area of $(z-1) / z$ in Figure 1, although part of this is also covered by the $1-z$ transformation. 
TABLE 3. Comparison of $1 /\left(z-z_{0}\right)$ algorithms in areas of interest. $|\mathcal{R}(a, b, c)| \leq 4,|\mathcal{I}(a, b, c)| \leq 4$ and not close to an integer; $10^{5}$ random draws for each entry.

\begin{tabular}{lcccc}
\hline \hline & \multicolumn{1}{c}{$t_{\mathrm{avg}}$} & $t_{\max }$ & $t_{\mathrm{avg}}$ & $t_{\max }$ \\
\hline & $|z-\exp (\pi \mathbf{i} / 3)| \leq 0.1$ & $|z-\exp (\pi \mathbf{i} / 3)| \leq 0.3$ \\
$A[1 /(2 z-1)]$ & 5.6 & 0.7 & 5.5 & 0.7 \\
$A[(z-1) /(z+1)]$ & 9.8 & 5.6 & 9.3 & 4.8 \\
$A[z /(z-1)-1 /(2 z)]$ & 9.8 & 5.6 & 9.4 & 4.8 \\
Balanced & 12.9 & 9.0 & 12.7 & 9.0 \\
\hline
\end{tabular}

When comparing $A[z /(z-1)-1 /(2 z)]$ to $A[1 /(2 z-1)]$ in more detail, we can make two interesting observations. Restricting ourselves to $\mathcal{R} z>0, A[z /(z-1)-1 /(2 z)]$ performs better closer to the circle of convergence, while $A[1 /(2 z-1)]$ performs better when further from the origin. Moreover, the former performs well for large $|d|=|c-a-b|$ or small $|\mathcal{I}(b-a)|$, and the latter for small $|d|$ or large $|\mathcal{I}(b-a)|$. So the two procedures are complimentary, and judicious selection between the two can improve the overall result. This is illustrated in Table 3 . In standard form, the three algorithms yield rather poor accuracy in circles around $\exp (\pi \mathbf{i} / 3)$. However, a balanced algorithm, which chooses between $A[z /(z-1)-1 /(2 z)]$ and $A[1 /(2 z-1)]$ on the basis of the parameters, shows excellent results.

It remains to fill in the blank space in Figure 1, and choose the best approach where the circles overlap. The easiest case is $\mathcal{R} z \leq 0$, which is almost entirely covered in the left panel of Figure 1. Outside $|z|=2.5$ there is essentially no difference between $A[1 / z]$ and $A[1 /(1-z)]$.

For $\mathcal{R} z>0$ we also use $A[1 / z]$ for larger $|z|$, but outside a tighter circle. Another thing to note is that $A[(z-1) / z]$ is used for $\mathcal{R} z \leq 0.4$ and $|z|<5$, provided $|\mathcal{I}(b-a)|$ is small: it is very effective there, and even marginally more accurate than $A[z]$ when $|z|<0.4$ (but a bit slower). Table 4 documents the algorithm $A\left[{ }_{2} F_{1}\right]$. No use is made of $A[1-z]$ and derived $A[1 /(1-z)]$, reducing complexity of $A\left[{ }_{2} F_{1}\right]$. It would be possible to replace $A[1 / z], A[(z-1) / z]$ by $A[1 /(2 z-1)], A[(z-1) /(z+1)]$ at a cost in speed.

Table 5 reports the accuracy of the proposed procedure 3 Note that in this table (and all results reported so far), $\mathcal{R}(a, b, c, c-a, c-b, c-a-b)$ have been kept away from integer values (positive as well as negative) by at least $10^{-2}$, by rejecting draws that are too close. This allows us to study the effect of the algorithm and the success of the removal of singularities separately. The results are based on $M=10^{6}$ random function evaluations.

The average accuracy of $A\left[{ }_{2} F_{1}\right]$ is about 13 digits throughout when considering moderate parameter values $|\mathcal{R}(a, b, c)| \leq 4$ and $|\mathcal{I}(a, b, c)| \leq 4$. Because the accuracy test requires $F(+2 ; z)$, the real (and imaginary) part of $d$ can range from -14 to 12 . The smaller worst-case accuracy, $t_{\max }$, only occurs in a tiny fraction of cases, because it has almost no impact on the average accuracy. As a comparison, we run

\footnotetext{
${ }^{3}$ The complex hypergeometric function has been implemented in double precision Ox code (see 4]), and partially duplicated in $\mathrm{C}++$. The accuracy tests and random number generation are done in Ox 7.0 .
} 
TABLE 4. Algorithm $A\left[{ }_{2} F_{1}\right], d=c-a-b$.

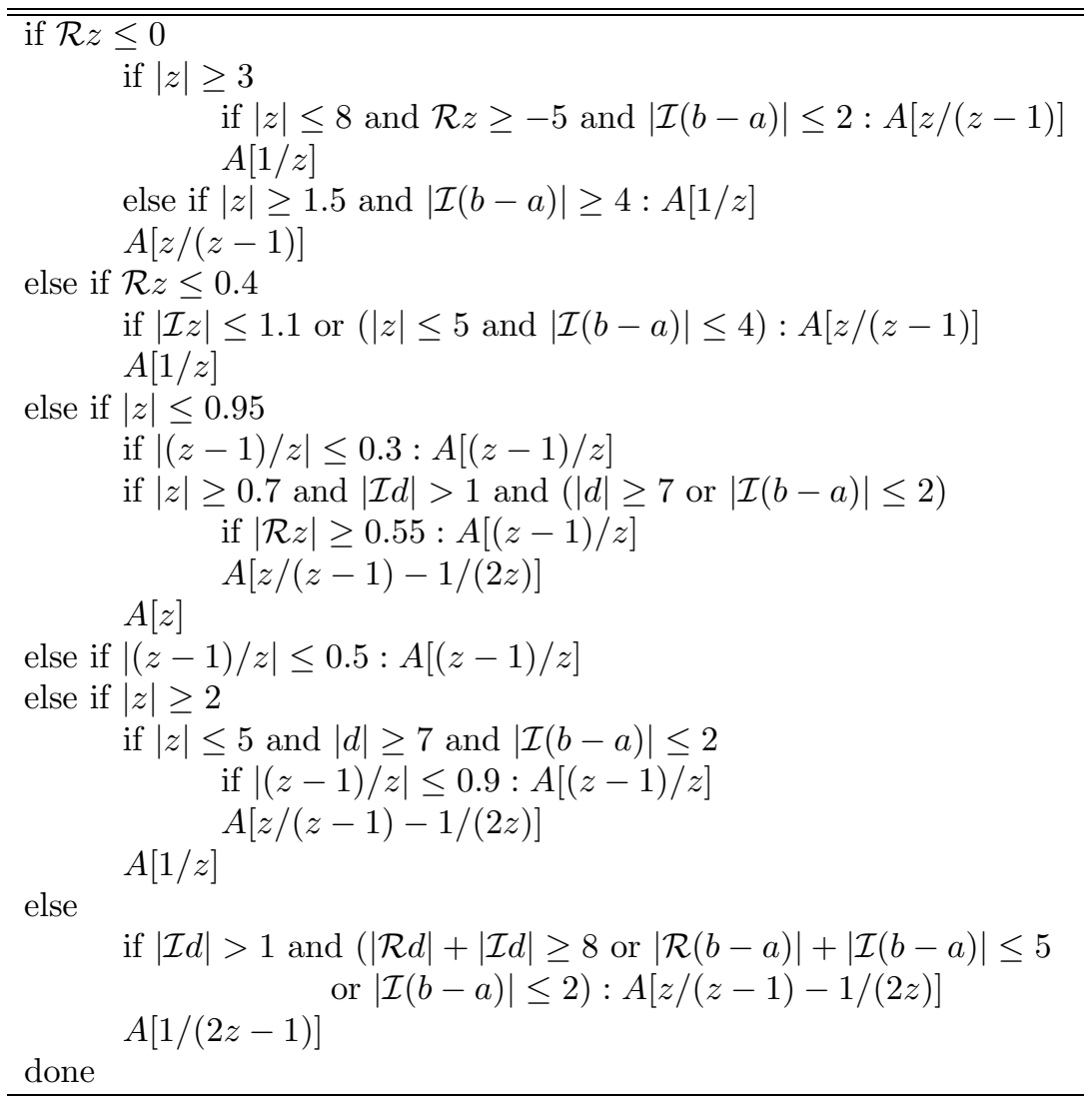

a similar procedure using the $\mathrm{C}++$ code of 9 . Both procedures were run on the same random draws, illustrating the improved accuracy of our procedure.

Behavior at or near singularity points is investigated separately. This is for two reasons. First, it is of interest to specify the distance from singularity explicitly, rather than leaving it to chance. The second reason is that standard random numbers, as generated by many algorithms, may not be able to reach the interesting parts of the parameter space. In particular, when the underlying random integers are 32-bit, then the uniform random numbers will live on a grid that is spaced by $2^{-32} \approx 2.3 \times 10^{-10}$. When sampling from $(-10,10)$, the grid becomes $\approx 4.6 \times 10^{-9}$; this may not be close enough to some integers. This issue (and an improvement, which is used in our random number generator) is discussed in [3].

The following modification to the randomly drawn parameters is adopted:

$$
\begin{array}{ll}
S_{1}: & \mathcal{R} a=\mathcal{R}(c-b-\lceil c-a-b\rfloor)+\varepsilon U_{1}, \mathcal{I} a=\mathcal{I}(c-b)-\varepsilon U_{2}, \\
S_{2}: & \mathcal{R} a=\mathcal{R}(b-\lceil b-a\rfloor)+\varepsilon U_{1}, \mathcal{I} a=\mathcal{I} b-\varepsilon U_{2},
\end{array}
$$

where $U_{i}$ is a uniform random number on $(-9,-1) \cup(1,9)$, and $\lceil\cdot\rfloor$ indicates rounding to the nearest integer: $\lceil x+\mathbf{i} y\rfloor=\lceil x\rfloor+\lfloor\mathbf{i} y\rfloor . S_{1}$ sets $d=c-a-b$ to a (near) integer, which is a singularity in $A[1-z], A[(z-1) / z], A[(z-1) /(z+1)]$ and $A[z /(z-1)-1 /(2 z)] . S_{2}$ sets $b-a$ to a (near) integer, which is a singularity in 
TABLE 5. Accuracy of proposed algorithm for ${ }_{2} F_{1}$ when $a, b, c, c-$ $a, c-b, c-a-b$ not close to an integer. Each case has $10^{6}$ random draws.

\begin{tabular}{|c|c|c|c|c|c|}
\hline & & $t_{\mathrm{avg}}$ & $t_{\max }$ & $t_{\mathrm{avg}}$ & $t_{\max }$ \\
\hline & & \multicolumn{4}{|c|}{$\mathcal{R}(a, b, c) \mid \leq 2$ and $|\mathcal{I}(a, b, c)| \leq 2$} \\
\hline & & \multicolumn{2}{|c|}{$A\left[{ }_{2} F_{1}\right]$ algorithm } & \multicolumn{2}{|c|}{ Michel \& Stoitsov } \\
\hline $0<\mathcal{R} z<1$ & $0<\mathcal{I} z<1$ & 14.9 & 11.1 & 13.4 & 8.4 \\
\hline $0<\mathcal{R} z<1$ & $-1<\mathcal{I}_{z}<0$ & 14.9 & 10.9 & 13.5 & 8.9 \\
\hline $1<\mathcal{R} z<2$ & $-1<\mathcal{I} z<1$ & 13.9 & 9.3 & 13.5 & 8.9 \\
\hline $0<\mathcal{R} z<2$ & $1<\mathcal{I} z<2$ & 13.5 & 9.5 & 13.3 & 8.9 \\
\hline$-2<\mathcal{R} z<0$ & $-2<\mathcal{I} z<2$ & 15.0 & 12.1 & 14.8 & 12.1 \\
\hline & & \multicolumn{4}{|c|}{$|\mathcal{R}(a, b, c)| \leq 4$ and $|\mathcal{I}(a, b, c)| \leq 4$} \\
\hline & & \multicolumn{2}{|c|}{$A\left[{ }_{2} F_{1}\right]$ algorithm } & \multicolumn{2}{|c|}{ Michel \& Stoitsov } \\
\hline $0<\mathcal{R} z<1$ & $0<\mathcal{I} z<1$ & 13.0 & 8.0 & 6.6 & 1.1 \\
\hline $0<\mathcal{R} z<1$ & $-1<\mathcal{I}_{z}<0$ & 13.1 & 8.2 & 6.1 & 0.6 \\
\hline $1<\mathcal{R} z<2$ & $-1<\mathcal{I} z<1$ & 12.6 & 8.1 & 4.7 & 0.0 \\
\hline $0<\mathcal{R} z<2$ & $1<\mathcal{I} z<2$ & 12.5 & 8.0 & 8.7 & 3.1 \\
\hline$-2<\mathcal{R} z<0$ & $-2<\mathcal{I} z<2$ & 13.9 & 9.4 & 10.7 & 5.7 \\
\hline
\end{tabular}

TABLE 6. Accuracy for $c-a-b, b-a$ close to integer, $|\mathcal{R}(a, b, c)| \leq$ $2,|\mathcal{I}(a, b, c)| \leq 2,0<\mathcal{R} z<2$ and $0<\mathcal{I} z<2$. Each pair of numbers in the table is for $S_{1}, S_{2}$, respectively (see text for a definition), $10^{5}$ draws.

\begin{tabular}{lccccccc}
\hline \hline \multicolumn{7}{c}{ Value of $\varepsilon$} \\
& $10^{-3}$ & $10^{-4}$ & $10^{-6}$ & $10^{-8}$ & $10^{-10}$ & $10^{-14}$ & $10^{-18}$ \\
\hline \multicolumn{7}{c}{$A\left[{ }_{2} F_{1}\right]$ algorithm } \\
$t_{\text {avg }}$ & 14,12 & 14,13 & 14,13 & 14,13 & 14,13 & 14,13 & 15,14 \\
$t_{\max }$ & 11,9 & 11,10 & 12,10 & 11,9 & 12,10 & 12,10 & 12,11 \\
\hline \multicolumn{7}{c}{ Michel \& Stoitsov } \\
$t_{\text {avg }}$ & 15,13 & 14,13 & 13,11 & 11,9 & 9,7 & 5,3 & 15,13 \\
$t_{\max }$ & 12,9 & 11,9 & 9,6 & 7,5 & 5,2 & 1,0 & 12,9 \\
\hline
\end{tabular}

$A[1 / z], A[1 /(1-z)]$ and $A[1 /(2 z-1)]$. There are no other integer conditions, so $c$ near a negative integer could also cause problems.

Table 6 presents the results for a range of $\varepsilon$ values; $\varepsilon=10^{-18}$ is below the machine precision. Each pair in the table is for $S_{1}, S_{2}$, respectively. The table shows that our procedure is not affected by the value of $\varepsilon$.

\section{Conclusions}

We presented an improved algorithm to evaluate the Gauss hypergeometric function. Effective solutions were provided for the singularities near integer values in some transformations. The expressions are cumbersome, but do work well in practice. Bühring's [2] transformation is often dismissed in the literature, but can now be seriously considered. We found that it does have a role to play in the current approach, but hope that it may eventually be replaced by much simpler expressions. 
Evaluation of ${ }_{2} F_{1}$ was extended to moderately large values of the arguments. Our next aim is to see if this can be extended further using other approaches, such as the continuation formulae studied in [7, and the interesting new series expansions derived in 8 .

\section{Appendix A. Removal of Singularities}

A.1. $1-z$ transformation, $d$ positive. Assume $\mathcal{R} d>0$ and write $d=c-a-b=$ $m+\epsilon$ with $m \geq 0$. So $m$ is the rounded real part of $d: m=\operatorname{round}(\mathcal{R} d)=\lceil\mathcal{R} d\rfloor=$ $\lfloor\mathcal{R} d+0.5\rfloor$, and $\epsilon=d-m$.

Starting from (8), the objective is to obtain a representation that can be evaluated accurately even for small $\epsilon$. Writing

$$
H=\Gamma\left(\begin{array}{c}
c,-m-\epsilon \\
a, b
\end{array}\right), J=\Gamma\left(\begin{array}{c}
c, m+\epsilon \\
b+m+\epsilon, a+m+\epsilon
\end{array}\right)
$$

for conciseness and collecting powers of $1-z$ :

$$
\begin{aligned}
& { }_{2} F_{1}(a, b ; c ; z)=H(1-z)^{m+\epsilon}{ }_{2} F_{1}(b+m+\epsilon, a+m+\epsilon ; 1+m+\epsilon ; 1-z) \\
& +J_{2} F_{1}(a, b ; 1-m-\epsilon ; 1-z) \\
& =H(1-z)^{m+\epsilon} \sum_{i=0}^{\infty} f_{i}(1-z)^{i}+J \sum_{i=0}^{\infty} g_{i}(1-z)^{i} \\
& =H(1-z)^{\epsilon} \sum_{i=0}^{\infty} f_{i}(1-z)^{m+i}+J \sum_{i=0}^{m-1} g_{i}(1-z)^{i}+J \sum_{i=m}^{\infty} g_{i}(1-z)^{i} \\
& =J \sum_{i=0}^{m-1} g_{i}(1-z)^{i}+H(1-z)^{\epsilon} \sum_{i=0}^{\infty} f_{i}(1-z)^{m+i}+J \sum_{i=0}^{\infty} g_{m+i}(1-z)^{m+i} \\
& =J \sum_{i=0}^{m-1} g_{i}(1-z)^{i}+\sum_{i=0}^{\infty}\left\{H(1-z)^{\epsilon} f_{i}+J g_{m+i}\right\}(1-z)^{m+i} \\
& =A_{1-z}^{+}+(1-z)^{m} B_{1-z}^{+} \text {. }
\end{aligned}
$$

$A_{1-z}^{+}=0$ when $m=0$; for $m>0$ there is no problem evaluating it for any $\epsilon$, because

$$
(1-m-\epsilon)_{i}=(1-m-\epsilon)(1-m-\epsilon+1) \ldots(1-m-\epsilon+i-1),
$$

where the last term is at most $(-1-\epsilon)$ when $i=m-1$. Starting from $g_{0}=1$ :

$$
g_{i}=\frac{(a+i-1)(b+i-1)}{(i-m-\epsilon)(m+i)} g_{i-1} .
$$

$B_{1-z}^{+}$cannot be evaluated as written for small $\epsilon$. Note, e.g., that

$$
g_{m+i}=\frac{(a)_{m+i}(b)_{m+i}}{(1-m-\epsilon)_{m+i}(m+i) !}=\frac{(a)_{m+i}(b)_{m+i}}{(1-m-\epsilon)_{m-1}(-\epsilon)(1-\epsilon)_{i}(m+i) !} .
$$

Start by writing

$$
B_{1-z}^{+}=\sum_{i=0}^{\infty} \beta_{i}(1-z)^{i}
$$


For the first term:

$$
\begin{aligned}
\beta_{0}= & H(1-z)^{\epsilon}+J \Gamma\left(\begin{array}{c}
1-m-\epsilon \\
1-\epsilon
\end{array}\right) \Gamma\left(\begin{array}{c}
a+m, b+m \\
a, b, m+1
\end{array}\right) \\
= & H(1-z)^{\epsilon}+J \Gamma\left(\begin{array}{c}
-m-\epsilon \\
1-\epsilon
\end{array}\right)(-m-\epsilon) \Gamma\left(\begin{array}{c}
a+m, b+m \\
a, b, m+1
\end{array}\right) \\
= & \frac{\epsilon \Gamma(-m-\epsilon)}{\epsilon \Gamma(1-\epsilon)} \Gamma\left(\begin{array}{c}
a+m, b+m \\
a, b, m+1
\end{array}\right) \\
& \times\left[\Gamma\left(\begin{array}{c}
c, m+1 \\
a+m, b+m
\end{array}\right)(1-z)^{\epsilon} \Gamma(1-\epsilon)-\Gamma\left(\begin{array}{c}
c, m+1+\epsilon \\
b+m+\epsilon, a+m+\epsilon
\end{array}\right)\right] .
\end{aligned}
$$

The numerator in the leading term can be written as

$$
\begin{aligned}
-\epsilon \Gamma(-m-\epsilon) & =\frac{\pi}{\sin \pi(-m-\epsilon)} \frac{1}{\Gamma(m+\epsilon)} \frac{-\epsilon}{-m-\epsilon} \\
& =(-1)^{m} \frac{\pi \epsilon}{\sin \pi \epsilon} \frac{1}{\Gamma(m+\epsilon+1)}
\end{aligned}
$$

giving

$$
\begin{aligned}
\beta_{0}= & (-1)^{m} \frac{\pi \epsilon}{\sin \pi \epsilon} \Gamma\left(\begin{array}{c}
c \\
a, b, m+\epsilon+1
\end{array}\right) \frac{1}{\epsilon} \\
& \times\left[\Gamma\left(\begin{array}{c}
a+m, b+m, m+1+\epsilon \\
a+m+\epsilon, b+m+\epsilon, m+1,1-\epsilon
\end{array}\right)-(1-z)^{\epsilon}\right]
\end{aligned}
$$

Writing $(1-z)^{\epsilon}=1+\epsilon E_{\epsilon}(\log [1-z])=1+\epsilon y$ and using

$$
h^{+}(\epsilon)=\Gamma\left(\begin{array}{c}
a+m, b+m, m+1+\epsilon \\
a+m+\epsilon, b+m+\epsilon, m+1,1-\epsilon
\end{array}\right)
$$

gives

$$
\begin{aligned}
\beta_{0} & =c_{0} \widetilde{\beta}_{0} \\
c_{0} & =\frac{(-1)^{m} \pi \epsilon}{\sin \pi \epsilon} \Gamma\left(\begin{array}{c}
c \\
a, b, m+\epsilon+1
\end{array}\right)=\frac{(-1)^{m}}{\operatorname{sinc}(\epsilon)} \Gamma\left(\begin{array}{c}
c \\
a, b, m+\epsilon+1
\end{array}\right), \\
\widetilde{\beta}_{0} & =\frac{h^{+}(\epsilon)}{\epsilon}-\frac{1}{\epsilon}[1+\epsilon y] \\
& =\frac{h^{+}(\epsilon)-1}{\epsilon}-y .
\end{aligned}
$$

When $|\epsilon| \geq 0.1$, the $\beta_{0}$ term is computed directly:

$$
\beta_{0}=\Gamma\left(\begin{array}{c}
c,-m-\epsilon \\
a, b
\end{array}\right)(1-z)^{\epsilon}+(-1)^{m} \Gamma\left(\begin{array}{c}
c, \epsilon \\
a+\epsilon, b+\epsilon, m+1
\end{array}\right) \frac{(a)_{m}(b)_{m}}{(a+\epsilon)_{m}(b+\epsilon)_{m}},
$$

otherwise we use (16)-(18) with

$$
\begin{aligned}
\widetilde{\beta}_{0}= & \left\{\left[-\Gamma_{-\epsilon}(a+m+\epsilon)\right] \Gamma\left(\begin{array}{c}
b+m \\
b+m+\epsilon
\end{array}\right)-\Gamma_{-\epsilon}(b+m+\epsilon)\right\} \Gamma\left(\begin{array}{c}
m+\epsilon+1,1 \\
m+1,1-\epsilon
\end{array}\right) \\
& +\Gamma_{\epsilon}(m+1) \Gamma\left(\begin{array}{c}
1 \\
1-\epsilon
\end{array}\right)+\Gamma_{\epsilon}(1-\epsilon)-y,
\end{aligned}
$$


where $y=0$ when $z=0$. The term inside curly braces is not expanded in $\Gamma_{-\epsilon}$ terms when $\left|\mathcal{R} \Gamma\left(\begin{array}{c}a+m, b+m \\ a+m+\epsilon, b+m+\epsilon\end{array}\right)-1\right| \geq 0.1$. Evaluation of $\Gamma_{\epsilon}(z)$ is discussed in $\oint \mathrm{B} .2$. The sinc function can also be evaluated accurately; see $\$ \mathrm{~B} .3$.

To compute the remaining $\beta_{i}$ it should be noted that subsequent terms add a division by $\epsilon$ which can be removed again. When $m>0$ :

$$
\begin{aligned}
\beta_{i+1} & =\Gamma\left(\begin{array}{c}
c,-m-\epsilon \\
a, b
\end{array}\right)(1-z)^{\epsilon} f_{i+1}+\Gamma\left(\begin{array}{c}
c, m+\epsilon \\
b+m+\epsilon, a+m+\epsilon
\end{array}\right) g_{m+i+1} \\
& =\Gamma\left(\begin{array}{c}
c,-m-\epsilon \\
a, b
\end{array}\right)(1-z)^{\epsilon} f_{i} \phi_{i+1}+\Gamma\left(\begin{array}{c}
c, m+\epsilon \\
b+m+\epsilon, a+m+\epsilon
\end{array}\right) g_{m+i} \gamma_{m+i+1} \\
& =\beta_{i} \phi_{i+1}+\Gamma\left(\begin{array}{c}
c, m+\epsilon \\
b+m+\epsilon, a+m+\epsilon
\end{array}\right) \epsilon g_{m+i}\left[\frac{\gamma_{m+i+1}-\phi_{i+1}}{\epsilon}\right] .
\end{aligned}
$$

The $\epsilon g_{m+i}$ term can be updated using (12). Then for the remainder 4

$$
\begin{aligned}
\frac{\gamma_{m+i+1}-\phi_{i+1}}{\epsilon} & =\frac{(a+m+i)(b+m+i)}{\epsilon(i+1-\epsilon)(m+i+1)}-\frac{(a+m+\epsilon+i)(b+m+\epsilon+i)}{\epsilon(m+\epsilon+i+1)(i+1)} \\
& =\frac{(1+i)(a-1)(b+m+i)+(m+1+i)[(b+m-1)(a+m+i)+\epsilon\{a+b-1+2 m+i+\epsilon\}]}{(i+1-\epsilon)(m+i+1)(m+\epsilon+i+1)(i+1)} .
\end{aligned}
$$

When $m=0$, the $\epsilon$ has to be taken out of the $\Gamma$ term:

$$
\beta_{i+1}=\beta_{i} \phi_{i+1}-\Gamma\left(\begin{array}{c}
c, 1+\epsilon \\
b+\epsilon, a+\epsilon
\end{array}\right) g_{i}\left[\frac{\gamma_{i+1}-\phi_{i+1}}{\epsilon}\right] .
$$

The $A_{1-z}^{+}$term is the same as in [11, eq. 3.27], but the $B_{1-z}^{+}$term there is expressed as a ratio of two infinite sums. [9, eq. 36] introduces a division by $\epsilon$ in $A_{1-z}^{+}$, is avoided here; 9 also handles the $\beta_{0}$ term somwehat differently.

A.1.1. Real arguments. We now assume that $a, b, c, z$ are real, so that ${ }_{2} F_{1}$ is real when $z<1$. If computational speed is important and we are interested in the real part of the answer, the algorithm can be implemented using real variables only. However, powers of negative real numbers must then be handled explicitly.

When a real expression involves real variables, except for a single power of a possibly negative real number $w$, we only need the real part:

$$
\mathcal{R} w^{a}= \begin{cases}w^{a} & w \geq 0 \\ \cos (\pi a)|w|^{a} & w<0 .\end{cases}
$$

This corresponds to $\lim _{\varepsilon \downarrow 0}(z+i \varepsilon)$. When there is a product of two powers of negative real numbers in the real expression, the sine terms will appear.

All previous expressions can be used when $z \leq 1$, because powers of negative real numbers are then absent. Otherwise, some adjustment is needed to capture the real part. First, we write

$$
(1-z)^{\epsilon}=[\cos (\pi \epsilon)+\mathbf{i} \sin (\pi \epsilon)]|1-z|^{\epsilon} .
$$

\footnotetext{
${ }^{4}$ For general $a, b, c, d$ we have

$$
\begin{aligned}
& (a+j)(b+j)(c+\epsilon+j)(d+j)-(a+\epsilon+j)(b+\epsilon+j)(c+j)(d-\epsilon+j) \\
& \quad=\epsilon[(a-c)(b+j)(d+j)+(b-d)(a+j)(c+j)]+\epsilon^{2}(c+j)[a+b-d+j]+\epsilon^{3}(c+j) .
\end{aligned}
$$
}


Then, writing $|1-z|^{\epsilon}=1+\epsilon y$, we only need the real part:

$$
\widetilde{\beta}_{0}=\frac{h^{+}(\epsilon)}{\epsilon}-\frac{1}{\epsilon}[1+\epsilon y] \cos (\pi \epsilon)=\frac{h^{+}(\epsilon)-1}{\epsilon}-\left[\frac{\cos (\pi \epsilon)-1}{\epsilon}+y \cos (\pi \epsilon)\right] .
$$

The additional term

$$
\frac{\cos (\pi \epsilon)-1}{\epsilon}=-\pi \sin (\pi \epsilon / 2) \operatorname{sinc}(\epsilon / 2)
$$

can be evaluated accurately; see $₫ \mathrm{~B} .3$.

In case transformation (5) is used to obtain $\mathcal{R} d>0$, the final result needs to be multiplied by $(1-z)^{-d}=(1-z)^{-m}(1-z)^{-\epsilon}$. In that case, and when $z>1$, the above results are insufficient, and we also need to obtain the sine component. Using (13):

$$
\begin{aligned}
\sin (\pi \epsilon) \Gamma\left(\begin{array}{c}
c,-m-\epsilon \\
a, b
\end{array}\right) \sum_{i=0}^{\infty} f_{i}(1-z)^{m+i} \\
\quad=(1-z)^{m} \Gamma\left(\begin{array}{c}
c, 1 \\
a, b, m+\epsilon+1
\end{array}\right)(-1)^{m}(-\pi) \sum_{i=0}^{\infty} f_{i}(1-z)^{i} \\
\quad=-|1-z|^{m}\left[\Gamma\left(\begin{array}{c}
c, 1 \\
a, b, m+\epsilon+1
\end{array}\right) \pi \sum_{i=0}^{\infty} f_{i}(1-z)^{i}\right]=-|1-z|^{m} C_{1-z}^{+} .
\end{aligned}
$$

The additional term is

$$
\left[\mathbf{i} \sin (-\pi d)|1-z|^{-d}\right]\left[\mathbf{i}|1-z|^{\epsilon}\left(-|1-z|^{m} C_{1-z}^{+}\right)\right]=-\sin (\pi d) C_{1-z}^{+} .
$$

The final expression, taking account of the transformation to $\mathcal{R} d>0$, is

$$
|1-z|^{-d} \cos (\pi d)\left[A_{1-z}^{+}+B_{1-z}^{+}\right]+C_{1-z}^{+} \sin (-\pi d) .
$$

A.2. $1 / z$ transformation. Assume $\mathcal{R} d>0$ and $d=b-a=m+\epsilon$ with $m \in \mathbb{N} \cup 0$.

Starting from (9), collecting powers of $w=1 / z$, and now writing

$$
H=\Gamma\left(\begin{array}{c}
c,-m-\epsilon \\
a, c-a-m-\epsilon
\end{array}\right), J=\Gamma\left(\begin{array}{c}
c, m+\epsilon \\
a+m+\epsilon, c-a
\end{array}\right),
$$

we find

$$
\begin{aligned}
& { }_{2} F_{1}(a, b ; c ; z)=H(-w)^{a+m+\epsilon}{ }_{2} F_{1}(a+m+\epsilon, 1-c+a+m+\epsilon ; 1+m+\epsilon ; w) \\
& \quad+J(-w)^{a}{ }_{2} F_{1}(a, 1-c+a ; 1-m-\epsilon ; w) \\
& =H(-w)^{a+m+\epsilon} \sum_{i=0}^{\infty} f_{i} w^{i}+J(-w)^{a} \sum_{i=0}^{\infty} g_{i} w^{i} \\
& =(-w)^{a} J \sum_{i=0}^{m-1} g_{i} w^{i}+(-w)^{a} \sum_{i=0}^{\infty}\left\{(-1)^{m}(-w)^{\epsilon} H f_{i}+J g_{m+i}\right\} w^{m+i} \\
& =(-w)^{a}\left(A_{1 / z}+w^{m} B_{1 / z}\right) .
\end{aligned}
$$


$A_{1 / z}$ can be evaluated as written. Expression (13) can be used for $B_{1 / z}$ :

$$
\begin{aligned}
& B_{1 / z}=\sum_{i=0}^{\infty} \beta_{i}^{r} w^{i} \\
& =H \sum_{i=0}^{\infty}\left\{(-1)^{m}(-w)^{\epsilon} \frac{1}{i !} \Gamma\left(\begin{array}{c}
a+m+\epsilon+i, 1-c+a+m+\epsilon+i, 1+m+\epsilon \\
a+m+\epsilon, 1-c+a+m+\epsilon, 1+m+\epsilon+i
\end{array}\right)\right. \\
& \left.-\Gamma\left(\begin{array}{c}
1, m+\epsilon+1 \\
a+m+\epsilon, c-a
\end{array}\right) \Gamma\left(\begin{array}{c}
a+m+i, 1-c+a+m+i, c-a-m-\epsilon \\
m+i+1,1-c+a, 1-\epsilon+i
\end{array}\right)\right\} w^{i} .
\end{aligned}
$$

Using

$$
\Gamma\left(\begin{array}{c}
1-c+a+m, c-a-m-\epsilon \\
1-c+a, c-a
\end{array}\right)=(-1)^{m} \Gamma\left(\begin{array}{c}
c-a-m-\epsilon \\
c-a-m
\end{array}\right)
$$

and introducing $b^{*}=c-a-m-\epsilon=c-b$ and

$$
h^{r}(\epsilon)=\Gamma\left(\begin{array}{c}
m+\epsilon+1, a+m, b^{*} \\
m+1, a+m+\epsilon, b^{*}+\epsilon, 1-\epsilon
\end{array}\right)
$$

together with $(-w)^{\epsilon}=1+\epsilon y$ we get

$$
\begin{aligned}
\beta_{0}^{r} & =c_{0}^{r} \widetilde{\beta}_{0}^{r}, \\
c_{0}^{r} & =\frac{1}{\operatorname{sinc} \epsilon} \Gamma\left(\begin{array}{c}
c \\
a, b^{*}, m+\epsilon+1
\end{array}\right), \\
\widetilde{\beta}_{0}^{r} & =\frac{h^{r}(\epsilon)}{\epsilon}-\frac{w^{-\epsilon}}{\epsilon} . \\
& =\frac{h^{r}(\epsilon)-1}{\epsilon}-y .
\end{aligned}
$$

Note that $h^{r}(\epsilon)$ equals $h^{+}(\epsilon)$ when $b^{*}-m$ is used instead of $b$ in (15).

Computing the remaining $\beta_{i}^{r}$ note again the similarity with $\$$ A.1. The recursions consist of:

$$
\begin{aligned}
\phi_{i+1} & =\frac{(a+m+\epsilon+i)(1-c+a+m+\epsilon+i)}{(m+\epsilon+i+1)(i+1)}, \\
\gamma_{m+i+1} & =\frac{(a+m+i)(1-c+a+m+i)}{(i+1-\epsilon)(m+i+1)} .
\end{aligned}
$$

So we can use the procedure for $\beta_{i}$ from $\$$ A.1 replacing $b$ there with $1-c+a$. This similarity with the $1-z$ case is not present in the approaches of [1] and [9].

When $|\epsilon| \geq 0.1$, direct evaluation is used for $\beta_{0}^{r}$.

For real arguments and $w>0$, the additional term is given by

$$
\sin (\pi a) w^{m+a+e} C_{1-z}^{+}(1-c+a),
$$

where $C_{1-z}^{+}(1-c+a)$ is (20), replacing $b$ with $1-c+a$.

A.3. $1 /\left(z-z_{0}\right)$ transformation. This transformation was obtained by Bühring [2], and removing the singularity is closely related to A.2.

Assume $d=b-a=m+\epsilon, \mathcal{R} d>0$ with $m \in \mathbb{N} \cup 0$. Starting from (10), using

$$
H=\Gamma\left(\begin{array}{c}
c,-m-\epsilon \\
a, c-a-m-\epsilon
\end{array}\right), J=\Gamma\left(\begin{array}{c}
c, m+\epsilon \\
a+m+\epsilon, c-a
\end{array}\right),
$$


and collecting powers of $w=1 /\left(z-z_{0}\right)$ :

$$
\begin{aligned}
& { }_{2} F_{1}(a, b ; c ; z)=H(-w)^{a+m+\epsilon} \sum_{i=0}^{\infty} f_{i}\left(z_{0}\right) w^{i}+J(-w)^{a} \sum_{i=0}^{\infty} g_{i}\left(z_{0}\right) w^{i} \\
& =(-w)^{a} J \sum_{i=0}^{m-1} g_{i}\left(z_{0}\right) w^{i}+(-w)^{a} \sum_{i=0}^{\infty}\left\{(-1)^{m}(-w)^{\epsilon} H f_{i}\left(z_{0}\right)+J g_{m+i}\left(z_{0}\right)\right\} w^{m+i} \\
& =(-w)^{a}\left(A_{1 /\left(z-z_{0}\right)}+w^{m} B_{1 /\left(z-z_{0}\right)}\right)=(-w)^{a}\left(A_{1 /\left(z-z_{0}\right)}+w^{m} \sum_{i=0}^{\infty} \beta_{i}^{b} w^{i}\right) .
\end{aligned}
$$

$A_{1 /\left(z-z_{0}\right)}$ can be evaluated using the three-term recurrence given in [2, eq. $65^{5}$ :

$$
\begin{aligned}
& g_{i}\left(z_{0}\right)=\frac{i+a-1}{i(i-m-\epsilon)} \\
& \quad\left[\left\{i-c+a+(1-2 i+m+\epsilon) z_{0}\right\} g_{i-1}\left(z_{0}\right)+z_{0}\left(1-z_{0}\right)(i+a-2) g_{i-2}\left(z_{0}\right)\right],
\end{aligned}
$$

with $g_{0}\left(z_{0}\right)=1$ and $g_{-1}\left(z_{0}\right)=0$. The equivalent expression for $f_{i}$ is:

$$
\begin{aligned}
f_{i}\left(z_{0}\right)= & \frac{i+a+m+\epsilon-1}{i(i+m+\epsilon)}\left[\left\{i-c+a+m+\epsilon+(1-2 i-m-\epsilon) z_{0}\right\} f_{i-1}\left(z_{0}\right)\right. \\
& \left.+z_{0}\left(1-z_{0}\right)(i+a+m+\epsilon-2) f_{i-2}\left(z_{0}\right)\right]
\end{aligned}
$$

The coefficients can also be expressed as hypergeometric functions in terms of $1 / z_{0}$ :

$$
\begin{aligned}
g_{i}\left(z_{0}\right)= & \frac{(a)_{i}(-1)^{i} z_{0}^{i}}{i !}{ }_{2} F_{1}\left(-i, 1+a-c, 1-m-\epsilon ; 1 / z_{0}\right) \\
= & \frac{(a)_{i}(1+a-c)_{i}}{(1-m-\epsilon)_{i} i !}+\sum_{j=0}^{i-1} \frac{(a)_{i}(-1)^{i}}{i !} \frac{(-i)_{j}(1+a-c)_{j}}{j !(1-m-\epsilon)_{j}} z_{0}^{i-j}, \\
f_{i}\left(z_{0}\right)= & \frac{(a+m+\epsilon)_{i}(-1)^{i} z_{0}^{i}}{i !}{ }_{2} F_{1}\left(-i, 1+a+m+\epsilon-c, 1+m+\epsilon ; 1 / z_{0}\right) \\
= & \frac{(a+m+\epsilon)_{i}(1+a+m+\epsilon-c)_{i}}{(1+m+\epsilon)_{i} i !}+ \\
& \sum_{j=0}^{i-1} \frac{(a+m+\epsilon)_{i}(-1)^{i}}{i !} \frac{(-i)_{j}(1+a+m+\epsilon-c)_{j}}{j !(1+m+\epsilon)_{j}} z_{0}^{i-j} .
\end{aligned}
$$

So we can write

$$
B_{1 /\left(z-z_{0}\right)}=\sum_{i=0}^{\infty} \beta_{i}^{b} w^{i}=\sum_{i=0}^{\infty} \beta_{i}^{r} w^{i}+\sum_{i=0}^{\infty} \delta_{i} w^{i},
$$

where $\beta_{i}^{r}$ is the same as in (22) - (24).

\footnotetext{
${ }^{5} d_{n}\left(a, z_{0}\right)$ there equals $g_{i}\left(z_{0}\right)$ here.
} 
To evaluate $\delta_{i}$, we need to collect powers of $z_{0}$ :

$$
\begin{aligned}
\delta_{i}= & (-1)^{m}(-w)^{\epsilon} H \sum_{j=0}^{i-1} \frac{(a+m+\epsilon)_{i}(-1)^{i}}{i !} \frac{(-i)_{j}(1+a+m+\epsilon-c)_{j}}{j !(1+m+\epsilon)_{j}} z_{0}^{i-j} \\
& +J \sum_{j=0}^{m+i-1} \frac{(a)_{m+i}(-1)^{m+i}}{(m+i) !} \frac{(-m-i)_{j}(1+a-c)_{j}}{j !(1-m-\epsilon)_{j}} z_{0}^{m+i-j} \\
= & z_{0}^{m} J \frac{(a)_{m+i}(-1)^{m+i}}{(m+i) !} \sum_{j=0}^{m-1} \frac{(-m-i)_{j}(1+a-c)_{j}}{j !(1-m-\epsilon)_{j}} z_{0}^{i-j} \\
& +(-1)^{m+i} \sum_{j=0}^{i-1}\left[(-w)^{\epsilon} H \frac{(a+m+\epsilon)_{i}}{i !} \frac{(-i)_{j}(1+a+m+\epsilon-c)_{j}}{j !(1+m+\epsilon)_{j}}\right. \\
& +J \frac{(a)_{m+i}}{(m+i) !} \frac{\left.(-m-i)_{m+j}(1+a-c)_{m+j}\right]}{(m+j) !(1-m-\epsilon)_{m+j}} z_{0}^{i-j} \\
= & A_{i}\left(z_{0}\right)+(-1)^{m+i} B_{i}\left(z_{0}\right)=A_{i}\left(z_{0}\right)+(-1)^{m+i} \sum_{j=0}^{i-1} \eta_{j}(i) z_{0}^{i-j} .
\end{aligned}
$$

The first term:

$$
\begin{aligned}
\eta_{0}(i) & =(-w)^{\epsilon} \Gamma\left(\begin{array}{c}
c,-m-\epsilon, a+m+\epsilon+i \\
a, c-a-m-\epsilon, a+m+\epsilon, i+1
\end{array}\right) \\
& +\Gamma\left(\begin{array}{c}
c, m+\epsilon, 1-m-\epsilon \\
a, m+1,1-\epsilon
\end{array}\right) \Gamma\left(\begin{array}{c}
a+m+i \\
a+m+\epsilon
\end{array}\right) \Gamma\left(\begin{array}{c}
1+a-c+m \\
1+a-c, c-a
\end{array}\right) \frac{(-m-i)_{m}}{(m+i) !}
\end{aligned}
$$

can be written a: 6

$$
\begin{aligned}
& \eta_{0}(i)=H \Gamma\left(\begin{array}{c}
a+m+\epsilon+i \\
a+m+\epsilon, i+1
\end{array}\right) \\
& \times\left[(-w)^{\epsilon}-\Gamma\left(\begin{array}{c}
c-a-m-\epsilon, m+1+\epsilon \\
c-a-m, m+1,1-\epsilon
\end{array}\right) \Gamma\left(\begin{array}{c}
a+m+i \\
a+m+i+\epsilon
\end{array}\right)\right] \\
& =H \Gamma\left(\begin{array}{c}
a+m+\epsilon+i \\
a+m+\epsilon, i+1
\end{array}\right)\left[(-w)^{\epsilon}-h^{r}(\epsilon) \frac{(a+m)_{i}}{(a+m+\epsilon)_{i}}\right] \\
& =H \frac{1}{i !}\left[(a+m+\epsilon)_{i}\left\{(-w)^{\epsilon}-h^{r}(\epsilon)\right\}+h^{r}(\epsilon)\left\{(a+m+\epsilon)_{i}-(a+m)_{i}\right\}\right] \text {. }
\end{aligned}
$$

Using $c_{0}^{r}$ and $\widetilde{\beta}_{0}^{r}$ from (21)-(24) gives

$$
\begin{aligned}
& \eta_{0}(i)=(-1)^{m} c_{0}^{r} \widetilde{\eta}_{0}(i) \\
& \widetilde{\eta}_{0}(i)=\frac{(a+m+\epsilon)_{i}}{i !} \widetilde{\beta}_{0}^{r}-\frac{(a+m+\epsilon)_{i}-(a+m)_{i}}{\epsilon i !} h^{r}(\epsilon)
\end{aligned}
$$

${ }^{6}$ Using:

$$
\Gamma\left(\begin{array}{c}
1+a-c+m \\
1+a-c, c-a
\end{array}\right)=(-1)^{m} \Gamma\left(\begin{array}{c}
1 \\
c-a-m
\end{array}\right) \text { and } \frac{-(m-i)_{m}}{(m+i) !}=(-1)^{m} \frac{1}{i !} .
$$


with $\epsilon$ in the last term canceling

$$
\begin{aligned}
& \frac{(a+m+\epsilon)_{0}-(a+m)_{0}}{\epsilon}=0, \\
& \frac{(a+m+\epsilon)_{1}^{\epsilon}-(a+m)_{1}}{\epsilon}=1, \\
& \frac{(a+m+\epsilon)_{i+1}-(a+m)_{i+1}}{\epsilon} \\
& \quad=(a+m+i) \frac{(a+m+\epsilon)_{i}-(a+m)_{i}}{\epsilon}+(a+m+\epsilon)_{i} .
\end{aligned}
$$

We only need the first two terms:

$$
\begin{aligned}
& \beta_{0}^{b}=\beta_{0}^{r}+A_{0}\left(z_{0}\right), \\
& \beta_{1}^{b}=\beta_{1}^{r}+\delta_{1}=\beta_{1}^{r}+A_{1}\left(z_{0}\right)-c_{0}^{r}\left[(a+m+\epsilon) \widetilde{\beta}_{0}^{r}-h^{r}(\epsilon)\right] z_{0},
\end{aligned}
$$

after which we can continue with a three-term recurrence:

$$
\begin{aligned}
\beta_{i+2}^{b}= & \beta_{i+1}^{b} \phi_{i+2}^{1}+\beta_{i}^{b} \phi_{i+2}^{0}+\Gamma\left(\begin{array}{c}
c, m+\epsilon \\
a+m+\epsilon, c-a
\end{array}\right) g_{m+i+1}\left(z_{0}\right)\left[\gamma_{m+i+2}^{1}-\phi_{i+2}^{1}\right] \\
& +\Gamma\left(\begin{array}{c}
c, m+\epsilon \\
a+m+\epsilon, c-a
\end{array}\right) g_{m}\left(z_{0}\right)\left[\gamma_{m+i+2}^{0}-\phi_{i+2}^{0}\right] .
\end{aligned}
$$

The $\phi$ and $\gamma$ terms are defined through:

$$
\begin{aligned}
& g_{i+2}\left(z_{0}\right)=\gamma_{i+2}^{1} g_{i+1}\left(z_{0}\right)+\gamma_{i+2}^{0} g_{i}\left(z_{0}\right), \\
& f_{i+2}\left(z_{0}\right)=\phi_{i+2}^{1} g_{i+1}\left(z_{0}\right)+\phi_{i+2}^{0} g_{i}\left(z_{0}\right) .
\end{aligned}
$$

Once again, $\epsilon / \epsilon$ can be removed analytically:

$$
\begin{aligned}
& \frac{\gamma_{m+i}^{0}-\phi_{i}^{0}}{\epsilon}=\frac{z_{0}\left(1-z_{0}\right)}{(m+i)(i-\epsilon) i(m+i+\epsilon)} \\
& \quad \times[\alpha(\alpha-1)(m+i+i)-(2 \alpha-1)(m+i) i-\epsilon(m+i)(i-2 \alpha+1-\epsilon)], \\
& \frac{\gamma_{m+i}^{1}-\phi_{i}^{1}}{\epsilon}=\frac{1}{(m+i)(i-\epsilon) i(m+i+\epsilon)}\left[i(m+i)\left(\alpha z_{0}-\delta-\alpha\left(1-z_{0}\right)\right)\right. \\
& \left.\quad+\alpha \delta(m+i+i)+\epsilon \alpha z_{0} i-\epsilon(m+i)\left\{\left(1-z_{0}\right) i-\delta-\alpha\left(1-z_{0}\right)-\left(1-z_{0}\right) \epsilon\right\}\right],
\end{aligned}
$$

where $\alpha=m+i+a-1$ and $\delta=m+i-c+a+(1-m-2 i) z_{0}$.

At the end of $A_{1 /\left(z-z_{0}\right)}$, we can do one more update to obtain $\epsilon g_{m}\left(z_{0}\right)$; then replacing $g_{m}\left(z_{0}\right)$ with $\epsilon g_{m}\left(z_{0}\right)$ gives the recursion for $\epsilon g_{m+i}\left(z_{0}\right)$. This also gives the $\epsilon$ that cancels out. When $m=0$, the $\epsilon$ is provided by the gamma term.

A.4. $(z-1) / z$ transformation. Starting from $(\underline{6}):{ }_{2} F_{1}(a, b ; c ; \bar{z})=(1-\bar{z})^{-a}{ }_{2} F_{1}(a$, $c-b ; c ; \bar{z} /[\bar{z}-1])$, we can use $\$$ A.2 with $z=\bar{z} /(\bar{z}-1)$. Now $w=1 / z=(\bar{z}-1) / \bar{z}$. When $a \leq c-b$ powers can be combined:

$$
(1-\bar{z})^{-a}(-w)^{a}=(1-\bar{z})^{-a}\left(\frac{1-\bar{z}}{\bar{z}}\right)^{a}=\bar{z}^{-a} .
$$

Otherwise

$$
(1-\bar{z})^{-a}(-w)^{c-b}=(1-\bar{z})^{c-a-b} \bar{z}^{b-c} .
$$


Similarly combining (6) with $\$$ A.3 gives the

$$
\frac{\bar{z}-1}{\bar{z}\left(1-z_{0}\right)+z_{0}}
$$

transformation.

\section{ApPEndix B. Evaluation of SUPPLEMENTARY FUnCTIONS}

B.1. Ratios of Gamma functions. The implementation of

$$
\Gamma\left(\begin{array}{l}
a, b \\
c, d
\end{array}\right)
$$

is quite involved:

(1) First equal arguments are removed:

$$
\Gamma\left(\begin{array}{c}
a, b \\
a, d
\end{array}\right) \rightarrow \Gamma\left(\begin{array}{c}
1, b \\
1, d
\end{array}\right)
$$

even for $a=0$.

(2) If all $\min \{\mathcal{R} a, \mathcal{R} b, \mathcal{R} c, \mathcal{R} d\}>10^{-6}$, then the function will return:

(a) the ratio of gamma functions if $\max \{\mathcal{R} a, \mathcal{R} b, \mathcal{R} c, \mathcal{R} d\}<50$, or

(b) the exponent of $\log \Gamma$ functions otherwise.

(3) The next stage is to detect pochhammer terms:

$$
\begin{aligned}
& \Gamma\left(\begin{array}{l}
a \\
b
\end{array}\right) \rightarrow(b)_{\lceil\mathcal{R}(a-b)\rfloor} \text { if } \mid(a-b)-\lceil\mathcal{R}(a-b)\rfloor<\epsilon_{p} b \text { and } \mathcal{R}(a-b) \geq 1 . \\
& \Gamma\left(\begin{array}{l}
a \\
b
\end{array}\right) \rightarrow\left[(a)_{-\lceil\mathcal{R}(a-b)\rfloor}\right]^{-1} \text { if } \mid(a-b)-\lceil\mathcal{R}(a-b)\rfloor<\epsilon_{p} a \text { and }-\mathcal{R}(a-b) \geq 1 .
\end{aligned}
$$

The pochhammer are evaluated in logarithms (with a separate sign for the real version).

(4) The remainder is evaluated with the $\log \Gamma$ function for each remaining term (say a) separately:

(a) if $\mathcal{R} a>10^{-8}$ directly; otherwise:

(b) if $\mid \mathcal{R} a-\left\lceil\mathcal{R} a||<10^{-7}\right.$ with a first order Taylor expansion; otherwise:

(c) if $|\mathcal{R} a-\lceil\mathcal{R} a\rfloor|<10^{-4}$ with a second order Taylor expansion; otherwise:

(d) directly with the $\log \Gamma$ function.

The Taylor expansion is split in a scale and a logarithmic term.

Finally, the answer is constructed through exponentiation.

When $\epsilon$ is small (but not too small), the ratio of two gamma functions close to a negative integer (but differently close) is computed as:

$$
\Gamma\left(\begin{array}{c}
-m+\epsilon \\
-k+\alpha \epsilon
\end{array}\right)=(-1)^{m-k} \alpha \frac{k !}{m !}+\mathcal{O}(\epsilon) .
$$

However, with $\epsilon$ sufficiently small (i.e., smaller than the machine precision):

$$
-k+\alpha \epsilon=-k, \text { and }-m+\epsilon=-m,
$$

so then:

$$
\Gamma\left(\begin{array}{c}
-m+\epsilon \\
-k+\alpha \epsilon
\end{array}\right)=\Gamma\left(\begin{array}{c}
-m \\
-k
\end{array}\right)=(-k)_{k-m}
$$


which does not correspond to the limit for $\epsilon \rightarrow 0$. This introduces some arbitrariness near this limit: $\alpha$ could be genuine (but imprecise), or just a result of a rounding error that occurred previously.

B.2. $\Gamma_{\epsilon}(z)$. To evaluate the function

$$
\Gamma_{\epsilon}(z)=\frac{1}{\epsilon}\left[\Gamma\left(\begin{array}{c}
z+\epsilon \\
z
\end{array}\right)-1\right]
$$

for small $\epsilon$ we adopt the Taylor expansion:

$$
\begin{aligned}
& \Gamma\left(\begin{array}{c}
z+\epsilon \\
z
\end{array}\right)=1+\epsilon \psi(z) \\
& \quad+\frac{\epsilon^{2}}{2}\left[\psi^{\prime}(z)+\psi(z)^{2}\right]+\frac{\epsilon^{3}}{6}\left[\psi^{\prime \prime}(z)+3 \psi^{\prime}(z) \psi(z)+\psi(z)^{3}\right]+\mathcal{O}\left(|\epsilon|^{4}\right) .
\end{aligned}
$$

So the digamma function $\psi(z)=\mathrm{d} \log \Gamma(z) / \mathrm{d} z$ and polygamma functions can be used for a finite difference approximation.

We can improve the approximation by one order of $\epsilon$ through central differences:

$$
\Gamma\left(\begin{array}{c}
z+\epsilon \\
z
\end{array}\right)-1=\left[\Gamma\left(\begin{array}{c}
z+\epsilon \\
z+\epsilon / 2
\end{array}\right)-\Gamma\left(\begin{array}{c}
z \\
z+\epsilon / 2
\end{array}\right)\right] \Gamma\left(\begin{array}{c}
z+\epsilon / 2 \\
z
\end{array}\right) .
$$

Write $z^{*}=z+\epsilon / 2$, then

$$
\Gamma_{\epsilon}(z)=\Gamma\left(\begin{array}{c}
z^{*} \\
z
\end{array}\right)\left[\psi\left(z^{*}\right)+\frac{(\epsilon / 2)^{2}}{6}\left[\psi^{\prime \prime}\left(z^{*}\right)+3 \psi^{\prime}\left(z^{*}\right) \psi\left(z^{*}\right)+\psi\left(z^{*}\right)^{3}\right]+\mathcal{O}\left(|\epsilon / 2|^{4}\right)\right] .
$$

To evaluate $\Gamma_{\epsilon}(z)$, we use (28) if $|\epsilon|<10^{-5} \min \{|z|, 1\}$, one additional term in the Taylor expansion if $|\epsilon|<10^{-2} \min \{|z|, 1\}$, and direct evaluation of (27) otherwise. If $\mathcal{R} z<0$, it is incremented, and the resulting ratio of Pochhammer terms written as $1+\epsilon g$ and multiplied out.

11] gives several alternative expessions for $\Gamma_{\epsilon}(z)$, which may be computationally more efficient.

B.3. $\operatorname{sinc}(z)$ and $\operatorname{cosc}(z)$.

$$
\begin{gathered}
\operatorname{sinc}(z)= \begin{cases}1 & z=0 \\
\frac{\sin (\pi z)}{\pi z} & |z|>0 .\end{cases} \\
\operatorname{cosc}(z)=\frac{1-\cos (\pi z)}{\pi z}=\sin \left(\frac{\pi z}{2}\right) \operatorname{sinc}\left(\frac{z}{2}\right) .
\end{gathered}
$$

B.4. $E_{\epsilon}(z)$.

$$
E_{\epsilon}(z)= \begin{cases}\sum_{k=1}^{\infty} \epsilon^{k-1} \frac{z^{k}}{k !} & |\epsilon z| \leq 0.02 \text { or }|z| \leq 0.02 \\ \frac{\exp (\epsilon z)-1}{\epsilon} & \text { otherwise. }\end{cases}
$$




\section{REFERENCES}

[1] M. Abramowitz and I. A. Stegun, Handbook of Mathematical Functions, Dover Publications Inc., New York, 1970.

[2] Wolfgang Bühring, An analytic continuation of the hypergeometric series, SIAM J. Math. Anal. 18 (1987), no. 3, 884-889, DOI 10.1137/0518066. MR883575 (88c:33004)

[3] J. A. Doornik, Conversion of high-period random numbers to floating point, ACM Transactions on Modeling and Computer Simulation 17 (2007).

[4] - Object-oriented Matrix Programming using Ox, 7th ed., Timberlake Consultants Press, London, 2013.

[5] Robert C. Forrey, Computing the hypergeometric function, J. Comput. Phys. 137 (1997), no. 1, 79-100, DOI 10.1006/jcph.1997.5794. MR1481885 (99g:33004)

[6] Amparo Gil, Javier Segura, and Nico M. Temme, The ABC of hyper recursions, J. Comput. Appl. Math. 190 (2006), no. 1-2, 270-286, DOI 10.1016/j.cam.2005.01.041. MR.2209508 (2006m:33003)

[7] , Numerically satisfactory solutions of hypergeometric recursions, Math. Comp. 76 (2007), no. 259, 1449-1468 (electronic), DOI 10.1090/S0025-5718-07-01918-7. MR.2299782 (2008d:33004)

[8] José L. López and Nico M. Temme, New series expansions of the Gauss hypergeometric function, Adv. Comput. Math. 39 (2013), no. 2, 349-365, DOI 10.1007/s10444-012-9283-y. MR.3082518

[9] N. Michel and M. V. Stoitsov, Fast computation of the Gauss hypergeometric function with all its parameters complex with application to the Pöschl-Teller-Ginocchio potential wave functions, Comput. Phys. Comm. 178 (2008), no. 7, 535-551, DOI 10.1016/j.cpc.2007.11.007. MR2585243

[10] A. B. Olde Daalhuis, Hypergeometric function, NIST Handbook of Mathematical Functions, Cambridge University Press, New York, 2010.

[11] S. L. Skorokhodov, A regularization method for computing the hypergeometric function $F(a, b ; c ; z)$ in a neighborhood of the singular points $z=1$ and $z=\infty$ (Russian, with Russian summary), Zh. Vychisl. Mat. Mat. Fiz. 41 (2001), no. 12, 1808-1832; English transl., Comput. Math. Math. Phys. 41 (2001), no. 12, 1718-1741. MR1882970 (2003k:33032)

[12] Nico M. Temme, Numerical aspects of special functions, Acta Numer. 16 (2007), 379-478, DOI 10.1017/S0962492906330012. MR2417932 (2009g:33027)

Institute for New Economic Thinking at the Oxford Martin School, University of OXFORD

E-mail address: jurgen.doornik@nuffield.ox.ac.uk 